\title{
Genetic Control and Host Range of Avirulence Toward Brassica napus Cultivars Quinta and Jet Neuf in Leptosphaeria maculans
}

\author{
M. H. Balesdent, A. Attard, D. Ansan-Melayah, R. Delourme, M. Renard, and T. Rouxel
}

First, second, and sixth authors: Pathologie Végétale, INRA, Route de Saint Cyr, F-78026 Versailles Cedex, France; third author: Service Biotechnologies, Aventis CropScience, Centre de Recherche de la Dargoire, 14-20, rue Pierre Baizet, F-69263 Lyon Cedex 09, France; and fourth and fifth authors: Amélioration des Plantes, INRA, Domaine de la Motte, BP 29, F-35653 Le Rheu Cedex, France.

Accepted for publication 18 September 2000.

\section{ABSTRACT}

Balesdent, M. H., Attard, A., Ansan-Melayah, D., Delourme, R., Renard, M., and Rouxel, T. 2001. Genetic control and host range of avirulence toward Brassica napus cultivars Quinta and Jet Neuf in Leptosphaeria maculans. Phytopathology 91:70-76.

Leptosphaeria maculans causes blackleg of oilseed rape. Gene-forgene interactions between race PG3 and Brassica napus cv. Quinta were related to interaction between the fungal avirulence (Avr) gene AvrLm1 and the corresponding resistance gene Rlm1. AvrLml isolates were avirulent on cvs. Doublol, Vivol, Columbus, and Capitol, and no recombinant phenotypes were observed in the progeny of two AvrLml $\times$ avrLml crosses, suggesting that all of these cultivars may possess $R \operatorname{lml}$ or genes displaying the same recognition spectrum, or that a cluster of Avr genes is present at the Avrlm1 locus. In one cross, segregation distortion was observed at the AvrLml locus that could be explained by interaction between AvrLml and one unlinked deleterious gene, termed Dell. Incompatibility toward cvs. Jet Neuf and Darmor.bzh was governed by a single gene, unlinked to AvrLm1 or Dell. This avirulence gene was termed AvrLm4. Preliminary plant genetic analysis suggested the occurrence of a corresponding dominant resistance gene, termed $R \operatorname{lm} 4$, present in the Quinta line analyzed and linked to Rlml.

Additional keywords: Phoma lingam.
The filamentous Ascomycete Leptosphaeria maculans (anamorph Phoma lingam) is the causal agent of blackleg of oilseed rape (Brassica napus L.). Blackleg is one of the most economically important disease of oilseed Brassicas worldwide $(16,30)$. Control of the pathogen can be achieved with fungicide treatments (9). However, their efficacy remains at a low level due to the lack of clear-cut forecasting schemes to optimize the use of fungicides (12). In consequence, genetic resistance remains the most environment friendly and reliable method of control. Genetic resistance to $L$. maculans is usually divided into adult-stage resistance and seedling resistance $(27,30)$. The former is the sum of many still uncharacterized general resistance mechanisms strongly interacting with the environment and growing conditions. Adult plant resistance is generally evaluated in the field by scoring symptoms at the end of the growing season. In contrast, seedling resistance is usually ascribed to single-gene race-specific resistance (2). Seedling resistance is easily scored following inoculations in controlled environment conditions, either on cotyledons or leaves $(1,2)$. Race-specific resistance aims at preventing the fungus from entering the plant, which is a prerequisite for biotrophic systemic growth within the plant that eventually leads to stem canker (17). In consequence, race-specific resistance is efficient to protect plants under field conditions whenever corresponding avirulent races are prevalent in local populations (3).

At present, two $B$. napus differential sets have been described $(5,18,26)$. They comprise either the three cvs. Westar, Quinta, and Glacier, which discriminates three pathogenicity groups (PG), termed PG2 to PG4, within the Tox ${ }^{+}$group of the L. maculans

Corresponding author: M. H. Balesdent; E-mail address: mhb@versailles.inra.fr

Publication no. P-2000-1018-01R

(c) 2001 The American Phytopathological Society species complex (14), or the four cvs. Lirabon, Quinta, Glacier, and Jet Neuf, discriminating six PGs termed A1 to A6 (Table 1). Even though genetic characterization of race-specific resistance in B. napus was described as early as 1980 (10), it is only recently that genetic control of a few specific interactions between $B$. napus and L. maculans was established on both the pathogen and the host plant; the avirulence (Avr) genes AvrLm1 and AvrLm2 in $L$. maculans are responsible for a specific resistance response in cvs. Quinta and Glacier, respectively $(1,2)$. The corresponding dominant resistance genes $R \operatorname{lm} 1$ and $R \operatorname{lm} 2$ were identified in cvs. Quinta and Glacier, respectively, providing the first evidence for a gene-for-gene relationship in the L. maculans-B. napus interaction (2). Another gene-for-gene interaction between the LEMI resistance locus in cv. Major and the Avr gene alm1 in isolate PHW1245 was reported $(11,28)$. To date, only a few additional sources for specific resistance have been identified in B. napus, and no other Avr locus has been described. Moreover, among the few major genetic loci operating at the seedling stage described in B. napus, no data are available regarding the possible allelism or redundancy of some of these genes, because genetic or quantitative trait loci studies were performed on seemingly unrelated cultivars, i.e., Major (11), Shiralee and Maluka (24), or Quinta and Glacier (2).

As a prerequisite to identify nonredundant resistance sources in B. napus, our objective is to genetically characterize races of the pathogen that will be used to (i) discriminate or identify resistance sources in different plant genetic backgrounds, and (ii) develop race-specific molecular markers for large-scale dynamic survey of the pathogen whenever novel resistance are used. In the present paper, we describe (i) the genetic characterization of AvrLm4 responsible for avirulent phenotype toward cv. Jet Neuf, (ii) linkage analysis of AvrLm4 with AvrLml, and (iii) preliminary data on genetic control of resistance to AvrLm4 isolates. 


\section{MATERIALS AND METHODS}

Fungal strains and culture maintenance. The a.2 (Mat ${ }^{+}$, AvrLml) and $\mathrm{H} 5$ (Mat' ${ }^{-}$avrLml) isolates of L. maculans were obtained from single ascospores isolated from diseased B. napus stubble $(1,15)$. Isolate 4.4.07 $\left(\mathrm{Mat}^{+}\right.$, avrLml) was obtained following in vitro crosses between one single-ascospore field isolate and one progeny of the cross a. $2 \times \mathrm{H} 5$ (22). Isolate IBCN5 is a single-ascospore isolate from the International Blackleg of Crucifers Network collection (29), formerly named $\mathrm{T} 11 \mathrm{aC} 25$ by the originator, H. H. Hoppe $(18,31)$. IBCN5 is a Mat', A5 isolate, i.e., avirulent on $B$. napus cv. Jet Neuf and virulent on the other genotypes of the differential set (Table 1) $(18,31)$.

All fungal cultures were maintained on V8-juice agar medium. Long-term storage was ensured by storing agar plugs collected from actively growing cultures in sterile mineral oil at $4^{\circ} \mathrm{C}$. For pathogenicity tests, 12-day-old sporulating cultures were obtained and conidia were collected as previously described (1).

In vitro crossing. In vitro crosses and tetrad or random ascospore recovery were performed according to Gall et al. (15) and Balesdent et al. (8). Progeny from in vitro crosses were named as follows: (i) in the case of tetrads, isolates are designated "i.j.kl" where "i" is the cross number, " $\mathrm{j}$ " refers to the pseudothecium from which spores are recovered, "k" refers to the ascus number, and "l" is a randomly attributed number from one to eight; and (ii) for random ascospores, isolates are termed "vi.j.k", where "i" and " $\mathrm{j}$ " refer to the cross number and pseudothecium number, respectively, and " $\mathrm{k}$ " is a randomly attributed number to distinguish isolates recovered from the same pseudothecium. Following tetrad recovery, the twin isolates within each tetrad were identified on the basis of the similarity of their electrokaryotypes, as previously established (22). Tetrads were considered complete when at least one isolate of each of the four genotypes of the tetrad was recovered. However, in most cases up to seven or eight isolates were recovered, allowing us to perform independent replicates of the inoculation tests by inoculating the twin isolates.

Ten tetrads and 88 random ascospores isolated from the a. $2 \times$ H5 cross (cross 11) $(1,2)$ were analyzed in the present study. In addition, seven complete tetrads were recovered from the cross IBCN5 $\times 4.4 .07$ (cross 21 ), and 89 random ascospores were recovered from the cross 21.3.01 × v11.3.6 (cross 23) (Table 2).

Plant material. Seeds of cvs. Westar, Glacier, and Quinta were provided by G. Séguin-Swartz (AAFC-SRC, Saskatoon, Canada), H. K. Love (Svalof Weibull Seed LTD, Lindsay, Canada), and H. H. Hoppe (Georg-August-Universität, Göttingen, Germany), respectively.

One plant of cv. Quinta displaying leaf resistance under field conditions $(2,3)$ was selected for selfing. For further multiplication, open pollination between a few selected S1 plants took place in the greenhouse. The resulting line was termed Quinta S2-V95. Line Quinta S1-3636 was obtained by selfing selected plants of the original seed lot cultivar. Additional selfings of individual plants produced five S2 lines: Quinta S2-V1 to S2-V4 and Quinta S2-4999. F2 and testcross populations from a cross between Quinta S1-3636 and plants of cv. Score were also used (2). The line Darmor.bz $h$ was obtained as described earlier (13). Seeds of the winter oilseed rape cvs. Vivol, Capitol, Doublol, and Columbus were obtained from Semences Cargill (Boissay, France).

Pathogenicity tests. Isolates were inoculated on cotyledons of B. napus as previously described (15). Four different isolates, i.e., one on each half-cotyledon were inoculated on each plant. At least 10 plants were inoculated with each isolate, and most experiments were repeated twice. Plants were incubated in a growth chamber at $16^{\circ} \mathrm{C}$ (night) and $-24^{\circ} \mathrm{C}$ (day) with a 12 -h photoperiod. Symptoms were scored 14 to 21 days after inoculation (dpi) with a modified Williams and Delwiche rating scale (34), where symptom scorings attributable to the Tox ${ }^{0}$ members of the $L$. maculans species complex $(7,14)$, i.e., infection classes (IC) IC2 and IC5 are omitted. This new scale, termed IMASCORE scale and described by Volke (31), comprises six IC, where IC1 is the typical hypersensitive response (HR), IC2 represents a larger (1.5 to $3 \mathrm{~mm}$ ) dark necrotic lesion, IC3 is a nonsporulating lesion that may or may not show tissue collapse as in IC4 to IC6, but that is always sharply delimited by a dark necrotic margin that may extend within the lesion. IC4 to IC6 are characterized by gray-green tissue collapse without a darkened margin, and showing no sporulation (IC4), a few pycnidia (IC5), or profuse sporulation (IC6). IC1 to IC3 are considered to be resistance responses, whereas IC4 to IC6 are considered to be susceptibility $(1,7)$.

\section{RESULTS}

AvrLm1 confers avirulence toward cvs. Quinta, Doublol, Vivol, Capitol, and Columbus. In cross 11, the AvrLml parental isolate a.2, which was described as avirulent on cv. Quinta and virulent on cvs. Westar and Glacier (1), was also avirulent on cvs. Doublol, Vivol, Capitol, and Columbus, whereas H5 was virulent on all of these cultivars (Table 3). Previous results showed that avirulence toward cv. Quinta segregates as a single trait in the 10 tetrads and 88 random ascospores of cross $11(1,2)$. Random progeny of cross 11 was further assessed for pathogenicity on Doublol, Vivol, Capitol, and Columbus. No recombinant phenotypes were recovered in the progeny, as all isolates that displayed the AvrLml phenotype (avirulence on cv. Quinta) were also avirulent on cvs. Doublol, Vivol, Capitol, and Columbus (Table 3). These data suggest that AvrLml, or genes tightly linked with AvrLml, is responsible for inducing resistance in cvs. Doublol, Vivol, Capitol, and Columbus. Because resistance to AvrLm1 isolates in cv. Quinta is due to the presence of the corresponding resistance gene Rlml (2), cvs. Doublol, Vivol, Capitol, and Columbus will be referred to as "Rlml-related cultivars" in the following text.

In the case of incompatible interactions on $R \operatorname{lm} 1$-related cultivars, the most frequently observed IC was IC2 (Fig. 1). Quinta S2-V95 and cv. Capitol could be distinguished from the other $R \operatorname{lm} 1$-related cultivars by the high frequency of IC3 (29.1 and $24.6 \%$, respectively, versus less than $10 \%$ for the other cultivars) (Fig. 1). In the case of the avrLml-Rlml (compatible) interactions, the most frequent class was IC4 or IC5, depending on the cultivar (Fig. 1). For instance, cv. Vivol was characterized by a slow development of susceptibility symptoms, with only $13.6 \%$ of sporulating lesions at $15 \mathrm{dpi}$ (IC5 and IC6), compared with more than $35 \%$ for the other Rlml-related cultivars (Fig. 1). Even in the case of compatible interactions, a significant percentage of resistance responses was observed at $15 \mathrm{dpi}$, because $6 \%$ of the $\approx 2,900$ inoculation sites observed belonged to IC1, IC2, or IC3 (Fig. 1). This was mainly the case for cvs. Columbus (9.7\%) and Vivol (19.7\%).

TABLE 1. Differential interactions expressed at the cotyledon stage between genotypes of Brassica napus and pathogenicity groups (PGs) of Leptosphaeria maculans

\begin{tabular}{|c|c|c|c|c|c|}
\hline L. $m a$ & aculans $^{\mathrm{a}}$ & B. nap & us genoty & pes $^{b}$ & \\
\hline Interaction 1 & Interaction 2 & Westar and Lirabon & Glacier & Quinta & $\overline{\text { Jet Neuf }}$ \\
\hline PG4 & $\begin{array}{l}\text { A1 } \\
\text { A5 }\end{array}$ & $\begin{array}{l}\mathrm{C} \\
\mathrm{C}\end{array}$ & $\mathrm{C}$ & $\begin{array}{l}\mathrm{C} \\
\mathrm{C}\end{array}$ & $\mathrm{C}$ \\
\hline PG3 & $\begin{array}{l}\mathrm{A} 2 \\
\mathrm{~A} 6\end{array}$ & $\begin{array}{l}\mathrm{C} \\
\mathrm{C}\end{array}$ & $\begin{array}{l}\mathrm{C} \\
\mathrm{C}\end{array}$ & $\begin{array}{l}\text { I } \\
\text { I }\end{array}$ & $\begin{array}{c}\text { C } \\
\text { I }\end{array}$ \\
\hline PG2 & $\begin{array}{l}\text { A4 } \\
\text { A3 }\end{array}$ & $\begin{array}{l}\mathrm{C} \\
\mathrm{C}\end{array}$ & $\begin{array}{l}\text { I } \\
\text { I }\end{array}$ & $\begin{array}{l}\text { I } \\
\text { I }\end{array}$ & $\begin{array}{c}\text { C } \\
\text { I }\end{array}$ \\
\hline
\end{tabular}

a Interaction 1 described by Mengistu et al. (26) and interaction 2 described by Hoppe et al. (18). Cvs. Westar, Glacier, and Quinta are described as differential according to Mengistu et al. (26), whereas Hoppe et al. (18) uses cv. Lirabon as susceptible control and cv. Jet Neuf as an additional differential. ${ }^{\mathrm{b}} \mathrm{C}=$ compatible interaction; $\mathrm{I}=$ incompatible interaction (resistance). 
Genetic control of avirulence toward cv. Jet Neuf. Incompatibility of IBCN5 toward cv. Jet Neuf, described by Hoppe et al. (18) was confirmed (Table 4, Fig. 2). In contrast to the AvrLm1Quinta incompatibility, the IBCN5-Jet Neuf interaction phenotype was a typical HR response, with only IC1 and IC2 (data not shown). IBCN5 also induced HR on Darmor.bzh, although it caused disease on the related cv. Darmor (Table 4). It was avirulent on Quinta lines S2-V95, S2-V1 to S2-V4, S1-3636, and S2-4999, whereas it was virulent on cvs. Doublol, Vivol, Capitol, Columbus, and the original Quinta seed lot (Table 4) (data not shown).

Incompatibility of IBCN5 toward cv. Jet Neuf segregated as a single trait in the progeny of cross 21 (Table 2), because half the genotypes within each tetrad behaved as IBCN5 (Table 4, Fig. 2). It is thus postulated that one $A v r$ gene, present in IBCN5, confers avirulence toward cv. Jet Neuf. All progeny of cross 21 that were avirulent on cv. Jet Neuf were also avirulent on Quinta S2-V95 and Darmor.bzh, but were virulent on the other cultivars (Table 4). The interaction phenotype of these isolates on Quinta S2-V95 was a typical HR (Fig. 3), clearly distinct from the late resistance response characteristic of the AvrLm1-Rlml interaction (Fig. 1) (3).

To further analyze the genetic linkage between AvrLml and the locus conferring avirulence toward cv. Jet Neuf, a cross was performed between isolate 21.3.01 (virulent on Rlml-related cultivars

TABLE 2. In vitro Leptosphaeria maculans crosses analyzed in this study

\begin{tabular}{lccllc}
\hline & \multicolumn{2}{c}{ Parental isolates } & & \multicolumn{2}{c}{ Progeny } \\
\cline { 2 - 3 } \cline { 6 - 7 } Cross & 1 & 2 & & Recovered & Selected \\
\hline 11 & a.2 & H5 & & 10 tetrads, 88 random ascospores & v11.3.06 \\
21 & IBCN5 & 4.4 .07 & & 7 tetrads & 21.3 .01 \\
23 & 21.3 .01 & v11.3.6 & & 89 random ascospores & \\
\hline
\end{tabular}

and avirulent on cv. Jet Neuf) and v11.3.6 (AvrLml and virulent on cv. Jet Neuf) (Table 5). The 89 random ascospore isolates recovered were inoculated on cvs. Westar, Glacier, Vivol, Doublol, Capitol, Columbus, and line Quinta S2-V95. All isolates were virulent on cvs. Glacier and Westar (data not shown). Based on a 55:45 avirulent/virulent ratio in the progeny, a single-gene control of avirulence toward cv. Jet Neuf was confirmed (chisquared $=0.91, \mathrm{df}=1, P>0.05$ ) (Table 5). Recombinant phenotypes, i.e., avirulent on both cv. Jet Neuf and Rlml-related cultivars or virulent on all cultivars, were obtained with a 20.22 and $28.10 \%$ frequency, respectively, which is compatible with the hypothesis that the locus conferring avirulence toward cv. Jet Neuf is unlinked to AvrLml (chi-squared $=0.1012$, $\mathrm{df}=1, P>$ 0.05) (Table 5). The locus responsible for avirulence toward cv. Jet Neuf was termed AvrLm4, and it is suggested that the genotypes of the parental isolates of cross 23 are avrLm1-AvrLm4, for isolate 21.3.01, and AvrLm1-avrLm4, for isolate v11.3.6 (Table 5).

However, as compared with cross 11, the segregation of AvrLm1 in cross 23 cannot be explained by a single gene, because a 63:37 virulent/avirulent segregation ratio is obtained at the AvrLm1 locus (chi-squared for a 50:50 expected ratio $=5.94$, df $=1, P<$ $0.05)$. One hypothesis to explain this segregation distortion could be the occurrence of a deleterious gene involved in cross 23, but not in cross 11, termed Dell, so that AvrLm1-Dell genotypes are not viable. Based on this hypothesis, the genotypes of parental isolates in cross 23 would be avrLm1-AvLm4-Dell (21.3.01) $\times$ AvrLm1-avrLm4-dell (v11.3.6) (Table 5). The observed avrLm1/ AvrLm1 ratio in cross 23 fits with the expected two-thirds/onethird ratio (chi-squared $=0.562, \mathrm{df}=1, P>0.05$ ). In addition, the four phenotypic classes in the progeny of cross 23 should be recovered with the expected ratios of two-sixths (avrLm1AvrLm4)/one-sixth (AvrLm1-avrLm4)/one-sixth (AvrLm1-AvrLm4)/

TABLE 3. Pathogenicity of the 88 random ascospore progeny of the Leptosphaeria maculans cross a.2 (AvrLm1) $\times$ H5 (avrLm1)

\begin{tabular}{|c|c|c|c|c|c|c|c|c|}
\hline \multirow[b]{2}{*}{ Isolates } & \multicolumn{8}{|c|}{ Brassica napus cultivar ${ }^{\text {a }}$} \\
\hline & Westar & Glacier & Quinta S2-V95 & Doublol & Vivol & Capitol & Columbus & Jet Neuf \\
\hline \multicolumn{9}{|l|}{ Parental isolates } \\
\hline a. 2 & $\mathrm{C}$ & $\mathrm{C}$ & I & I & I & I & I & $\mathrm{C}$ \\
\hline H5 & $\mathrm{C}$ & $\mathrm{C}$ & $\mathrm{C}$ & $\mathrm{C}$ & $\mathrm{C}$ & $\mathrm{C}$ & $\mathrm{C}$ & $\mathrm{C}$ \\
\hline \multicolumn{9}{|c|}{ Interaction phenotypes in progeny } \\
\hline 1 (43 isolates) & $\mathrm{C}$ & $\mathrm{C}$ & I & I & I & I & I & nd \\
\hline 2 (45 isolates) & $\mathrm{C}$ & $\mathrm{C}$ & $\mathrm{C}$ & $\mathrm{C}$ & $\mathrm{C}$ & $\mathrm{C}$ & $\mathrm{C}$ & nd \\
\hline
\end{tabular}

a $\mathrm{C}=$ compatible interaction; $\mathrm{I}=$ incompatible interaction; $\mathrm{nd}=$ not determined.
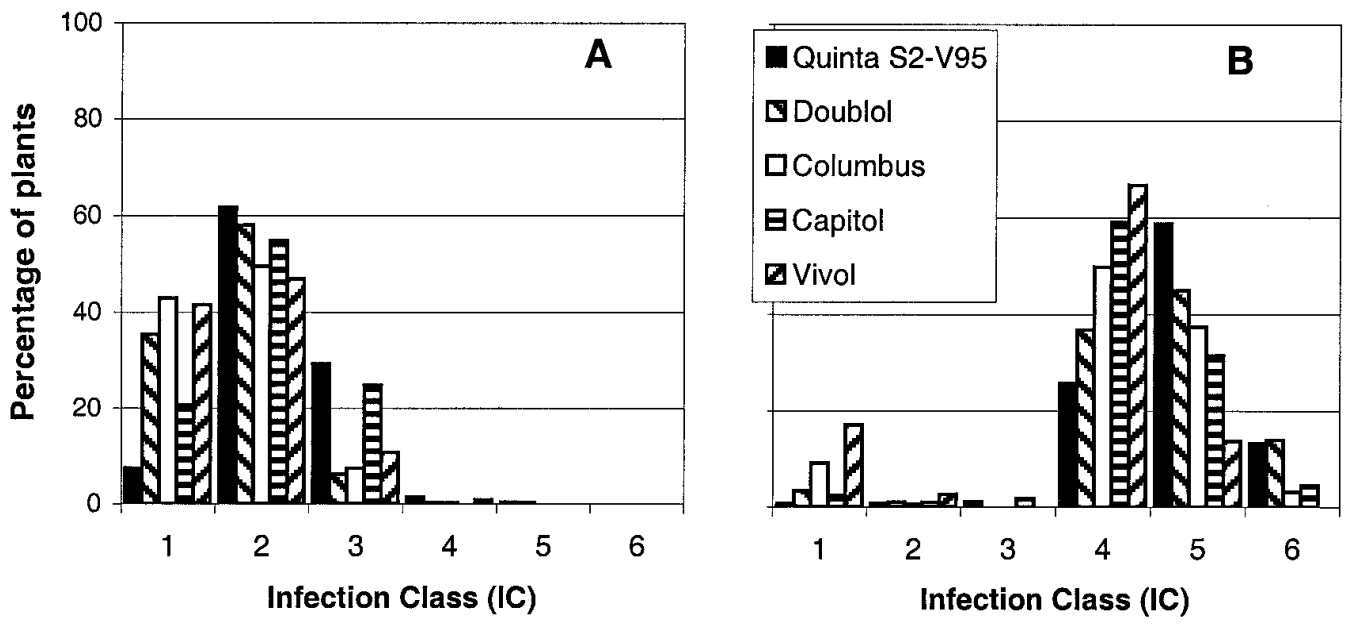

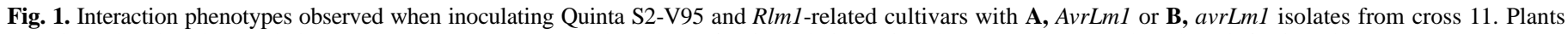

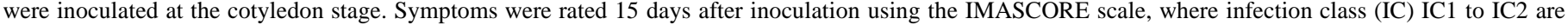

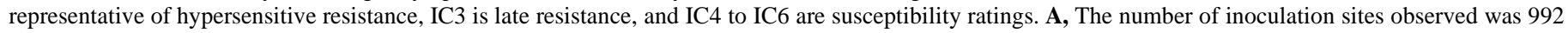

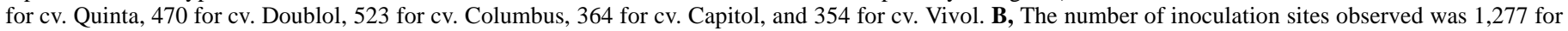
cv. Quinta, 503 for cv. Doublol, 546 for cv. Columbus, 293 for cv. Capitol, and 264 for cv. Vivol. 
two-sixths (avrLm1-avrLm4). The observed ratio for these four classes (Table 5) support the hypothesis (chi-squared $=1.4735$, $\mathrm{df}=3, P>0.05$ ). Finally, as in cross 11 , no recombinant phenotypes were observed when considering the set of Rlml-related cultivars (Table 5).

Preliminary assessment of genetic control of resistance to AvrLm4. The occurrence of the dominant resistance gene Rlml, interacting with AvrLml, was previously demonstrated following genetic analyses of F1, F2, and testcross populations of Quinta $\times$ Score crosses (2). The Quinta line used in these crosses was resistant toward AvrLm4 isolates, whereas cv. Score was susceptible to AvrLm4 isolates (Table 6). Consequently, the F2 and testcross progeny of Quinta $\times$ Score crosses were used to analyze the genetic control of the resistance of cv. Quinta to isolates harboring AvrLm4. F2 and testcross plants were inoculated with isolates v11.1.2 (AvrLm1-avrLm4) and v23.2.1 (avrLm1-AvrLm4). Genefor-gene interaction between AvrLml and Rlml in cv. Quinta (2) was confirmed in both F2 and testcross populations, because the segregation ratio of the resistance to v11.1.2 was highly significant of a 1:1 and 3:1 resistant to susceptible ratio in testcross and F2 populations, respectively (chi-squared $=0.277$ and 0.891 , respectively, $\mathrm{df}=1, P>0.05$ ) (Table 6). Similar results were obtained when considering the segregation of resistance of Quinta S1-3636 to v23.2.1, because a 98:79 resistant to susceptible ratio in testcross, and a 127:38 resistant to susceptible ratio in the F2 (chisquared $=2.04$ and 0.341 , respectively, for a $1: 1$ and 3:1 ratio, $\mathrm{df}=1, P>0.05)$ were obtained. It is thus suggested that a

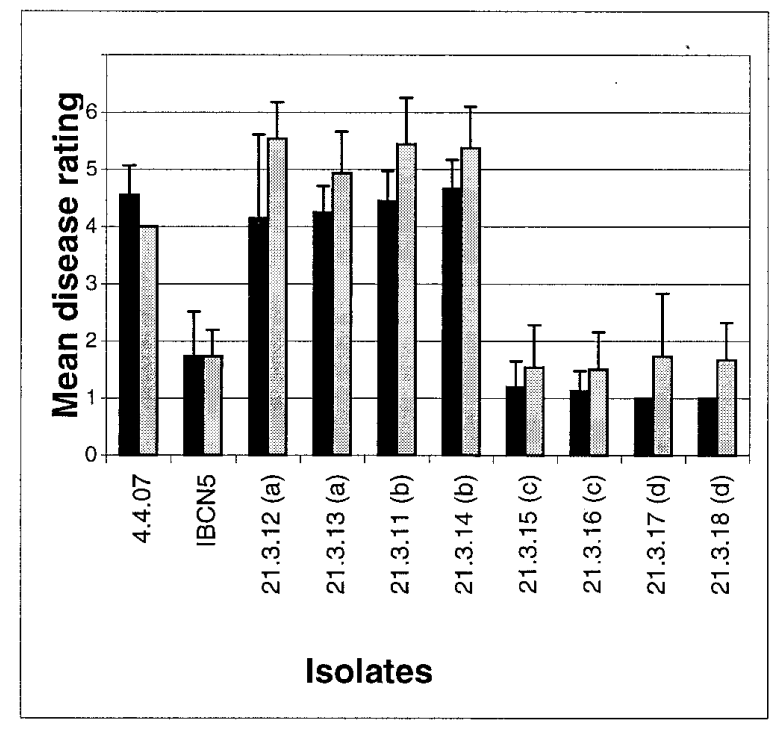

Fig. 2. Example of the segregation of interaction phenotypes on cvs. Jet Neuf (black) and Quinta S2-V95 (gray). Symptoms were rated 15 days after inoculation using the IMASCORE scale. 4.4.07 and IBCN5, parental isolates of cross 21; other isolates are members of one tetrad resulting from this cross. Twin genotypes within the tetrad share the same letter between parentheses.

TABLE 4. Characterization of the progeny of a cross between two Leptosphaeria maculans isolates differing in their interaction phenotype on Brassica napus cv. Jet Neuf

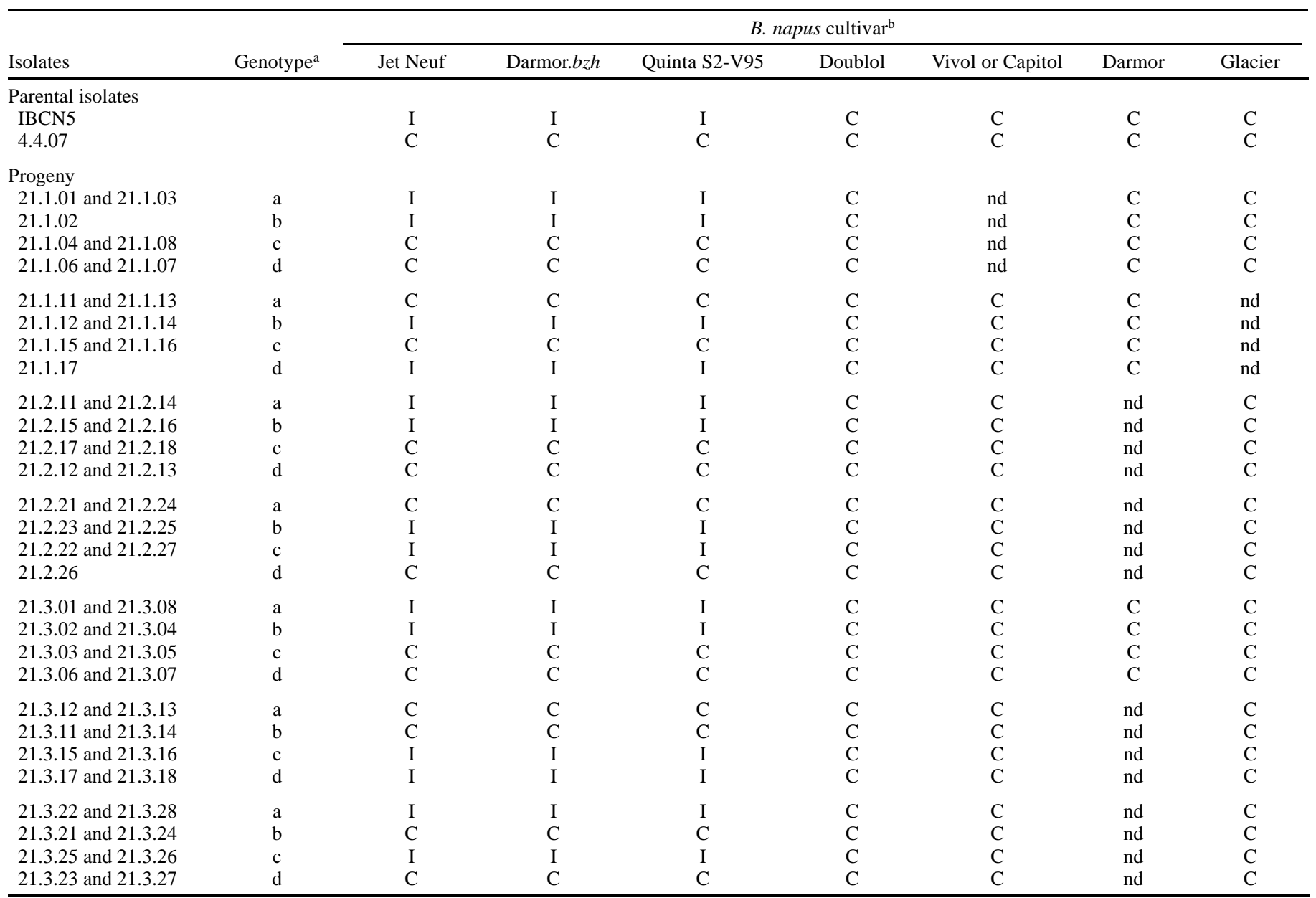

${ }^{a}$ Genotypes (a to d) within each tetrad were identified following pulsed-field gel electrophoresis of whole chromosomes (22). All isolates of the progeny between each a to $\mathrm{d}$ belong to the same tetrad.

${ }^{\mathrm{b}} \mathrm{C}=$ compatible interaction; $\mathrm{I}=$ incompatible interaction; $\mathrm{nd}=$ not determined. 
dominant resistance gene conferring resistance to AvrLm4 is present in the Quinta line used in the crosses. The occurrence of recombinant phenotypes in both $\mathrm{F} 2$ and testcross populations (Table 6) suggests that the locus responsible for resistance to AvrLm4 is not Rlm1. The dominant resistance gene recognizing AvrLm4 was designated Rlm4. The observed ratio of plants in each of the four phenotypic classes (Table 6) suggests a genetic linkage between $R \operatorname{lm} 1$ and $R \operatorname{lm} 4$ (chi-squared $=26.89$ and 10.53 for testcross and $\mathrm{F} 2$, respectively, $\mathrm{df}=1, P<0.05$ ).

\section{DISCUSSION}

A gene-for-gene relationship was established between AvrLm1 and the corresponding resistance gene, Rlml, in cv. Quinta, and between AvrLm 2 and the corresponding resistance gene, $R \operatorname{lm} 2$, in cv. Glacier (2). In this paper, we report on the genetic identification of a novel avirulence gene, AvrLm4, specifying incompatibility on cv. Jet Neuf. The cross between one AvrLml and one AvrLm4 isolate generated a progeny comprising 89 isolates, among which the ratio of recombinant genotypes is highly indicative of genetic independence between AvrLml and AvrLm4. This data, along with previously demonstrated genetic linkage between AvrLml and AvrLm2 (2), indicates that at least two independent genomic regions of $L$. maculans are involved in avirulence toward B. napus. The AvrLm4 region specifies incompatibility toward cv. Jet Neuf, whereas the AvrLm1-AvrLm2 region specifies incompatibility

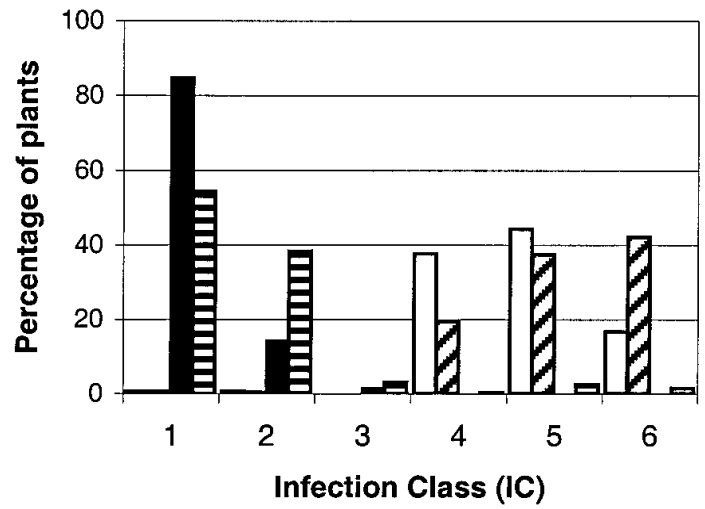

Fig. 3. Interaction phenotypes observed when inoculating Quinta S2-V95 and cv. Jet Neuf with progeny of cross 21 . White bar, isolates virulent on cv. Jet Neuf inoculated on cv. Jet Neuf; oblique stripes, isolates virulent on Quinta S2-V95 inoculated on Quinta S2-V95; black bars, isolates avirulent on cv. Jet Neuf inoculated on cv. Jet Neuf; horizontal bars, the same isolates, inoculated on Quinta S2-V95. Plants were inoculated at the cotyledon stage. Symptoms were rated 16 to 17 days after inoculation with the IMASCORE scale. The data synthesized 170 ratings in the case of cv. Jet Neuf and 325 ratings for Quinta S2-V95. toward cvs. Glacier, Quinta (2), and Rlm1-related cultivars. These linkage data are consistent with the occurrence of races A2, A5, and A6 (Table 1) in natural populations (5), whereas field isolates avirulent toward cv. Glacier and virulent toward cv. Quinta have not been described $(2,5,19,21,21,23,26)$. In addition, the lack of recombinant phenotypes in the 10 tetrads and 177 random progeny of two AvrLml $\times$ avrLml crosses, when inoculated on the set of cvs. Quinta, Doublol, Vivol, Capitol, and Columbus, suggested either that these cultivars all possess $R \operatorname{lml}$ or resistance genes displaying the same recognition spectrum, or that a cluster of tightly linked $A v r$ genes is present in the AvrLml region. Such Avr gene clusters have been described in two other plant-fungus interactions, i.e., Mycosphaerella graminicola-wheat and Phytophthora sojae-soybean $(32,33)$. Finally, segregation distortion at the AvrLm1 locus was observed in some, but not all, crosses, and could be explained by a lethal interaction between AvrLm1 and one gene, termed Dell, unlinked to AvrLml or to AvrLm4. This hypothesis, that still needs to be confirmed, implies that Dell could exert a counter-selection facilitating the dissemination of avrlml isolates, therefore reducing the durability of $R \operatorname{lm} 1$ resistance.

The genetic evidence for a new avirulence gene, AvrLm4, suggested the occurrence of a corresponding resistance gene, that reacts to AvrLm4 isolates, in cv. Jet Neuf and in some Quinta lines. Plant genetic studies indicate the occurrence of a single gene control of resistance to AvrLm4 in the Quinta line used. This resistance locus, termed $R \operatorname{lm} 4$, was found to be distinct from, but linked to, $R \operatorname{lm} 1$. It can be hypothesized that $R \operatorname{lm} 4$ is also present in Quinta S2-V95 and cvs. Jet Neuf and Darmor.bzh. This result is in accordance with genetic studies performed by Delwiche (10) on breeding line R39, which later became cv. Jet Neuf.

TABLE 6. Segregation of resistance to Leptosphaeria maculans isolates harboring AvrLm4 or AvrLm1 in Brassica napus line Quinta S1-3636

\begin{tabular}{lcrrrr}
\hline & & \multicolumn{4}{c}{ Plants per phenotypic class ${ }^{\mathrm{a}}$} \\
\cline { 5 - 6 } Isolate & Plants analyzed & S-S & S-R & R-S & R-R \\
\hline Parental lines & & & & & \\
$\quad$ Quinta & 156 & 0 & 0 & 0 & 156 \\
$\quad$ Score & 36 & 36 & 0 & 0 & 0 \\
$\begin{array}{l}\text { Quinta } \times \text { Score progeny } \\
\text { Testcross }\end{array}$ & 177 & 55 & 24 & 30 & 68 \\
$\quad$ F2 & 165 & 16 & 22 & 20 & 107 \\
$\quad$ Control cultivars & 25 & 0 & 0 & 25 & 0 \\
$\quad$ Jet Neuf & 23 & 0 & 22 & 0 & 1 \\
$\quad$ Columbus & 23 &
\end{tabular}

${ }^{a}$ Each plant was inoculated with isolates v23.2.1 (avrLm1-AvrLm4) and v11.1.2 (AvrLm1-avrLm4). The interaction phenotypes were scored 14 days after inoculation. Each plant was classified in one of four interaction phenotype classes: S-S (susceptibility to both isolates), S-R (susceptibility to v23.2.1, resistance to v11.1.2), R-S (resistance to v23.2.1, susceptibility to v11.1.2), and R-R (resistance to both isolates).

TABLE 5. Segregation analysis of interaction phenotypes in a cross between two Leptosphaeria maculans isolates differing in their interaction phenotypes on cv. Jet Neuf and Rlml-related cultivars

\begin{tabular}{|c|c|c|c|c|c|c|c|}
\hline \multirow[b]{2}{*}{ Isolates } & \multicolumn{3}{|c|}{ Brassica napus cultivars ${ }^{\mathrm{a}}$} & \multirow[b]{2}{*}{$\begin{array}{l}\text { No. of } \\
\text { isolates }\end{array}$} & \multirow[b]{2}{*}{$\begin{array}{l}\text { Observed } \\
\text { ratio }\end{array}$} & \multirow[b]{2}{*}{$\begin{array}{l}\text { Expected ratio and } \\
\text { putative genotype }^{b}\end{array}$} & \multirow[b]{2}{*}{$\begin{array}{l}\text { Expected ratio and } \\
\text { putative genotype }\end{array}$} \\
\hline & $\begin{array}{c}\text { Capitol, Vivol } \\
\text { Columbus, Doublol }\end{array}$ & Jet Neuf & Quinta S2-V95 & & & & \\
\hline \multicolumn{8}{|c|}{ Parental isolates } \\
\hline 21.3.01 & $\mathrm{C}$ & I & I & $\ldots$ & $\ldots$ & avrLm1 AvrLm4 & avrLm1 AvrLm4 Del1 \\
\hline v11.3.6 & $\mathrm{I}$ & $\mathrm{C}$ & $\mathrm{I}$ & $\ldots$ & $\ldots$ & AvrLm1 avrLm4 & AvrLm1 avrLm4 del1 \\
\hline \multicolumn{8}{|c|}{$\begin{array}{l}\text { Interaction phenotypes } \\
\text { in progeny }\end{array}$} \\
\hline 1 & $\mathrm{C}$ & I & I & 31 & $34.83 \%$ & 1/4, avrLm1 AvrLm4 & 2/6, avrLm1 AvrLm4 dell or Del1 \\
\hline 2 & I & $\mathrm{C}$ & $\mathrm{I}$ & 15 & $16.86 \%$ & 1/4, AvrLm1 avrLm4 & 1/6, AvrLm1 avrLm4 del1 \\
\hline 3 & I & I & $\mathrm{I}$ & 18 & $20.22 \%$ & 1/4, AvrLm1 AvrLm4 & 1/6, AvrLm1 AvrLm4 dell \\
\hline 4 & $\mathrm{C}$ & $\mathrm{C}$ & $\mathrm{C}$ & 25 & $28.09 \%$ & 1/4, avrLm1 avrLm4 & 2/6, avrLm1 avrLm4 dell or Del1 \\
\hline
\end{tabular}

${ }^{\mathrm{a}} \mathrm{C}=$ compatible interaction; $\mathrm{I}=$ incompatible interaction.

b Expected ratio and putative genotypes for two unlinked Avr genes, AvrLm1 and AvrLm4.

c Expected ratio and putative genotypes for two unlinked Avr genes (AvrLm1 and AvrLm4) and one deleterious (Dell) gene interacting with AvrLm1. 
An increasing number of reports indicate that gene-for-gene incompatibility is common in the L. maculans $-B$. napus interaction $(2,11,28)$. However, although very numerous specific interactions have been described (20), only few of them were submitted to genetic analysis. One prerequisite for developing genetic studies would be a standardized differential set using well-defined cultivars or DH lines, with characterized resistance genes (28). At present, as exemplified in the current study with cv. Quinta, major inconsistencies can be obtained when using different lines issued from the same cultivar. This is probably due to the heterogeneous nature of the B. napus cultivars grown in most parts of the world, as only a few countries require registration of pure lines. At least three different lines can be originated from cv. Quinta, harboring (i) Rlm1, (ii) Rlml and Rlm4 (this study), or (iii) none of these resistance genes $(3,19)$. In consequence, depending on the Quinta line used in the differential set comprising cvs. Westar or Lirabon, Quinta, Glacier, and Jet Neuf, one will be able to discriminate six PGs, if the Quinta line does not harbor $\operatorname{Rlm} 4(5,18)$ (Table 1), but only three PGs if the Quinta line harbors Rlm1 and Rlm4. In the latter case, it is impossible to state whether PG3 isolates possess only AvrLml (A2 isolates), AvrLm4 (A5 isolates), or both Avr genes (A6 isolates) (Table 1). Even when using seed of the original Quinta cultivar, interpretation of the outcome of the interaction with AvrLml isolates may be complicated by (i) the intermediate rating (IC3) usually resulting from this incompatibility reaction and (ii) the possibly high ratio of rlml plants, susceptible to AvrLml isolates (3). Both intermediate resistance and susceptibility ratings can result in an average rating indicative of susceptibility rather than incompatibility and of an erroneous interpretation of the data. In this case, the analysis should be focused on the ratio of resistant to susceptible plants rather than on the average disease index. In the present study, one Rlml-related cultivar, Columbus displayed highly consistent responses either to AvrLm1 or to avrLml isolates, as compared with other Rlml-related cultivars. Replacement of cv. Quinta by cv. Columbus could be a first step toward the standardization of the differential set.

The data presented here suggest that, due to the heterogeneity of some $B$. napus cultivars and the need for large-scale inoculation tests to determine unequivocally the outcome of one given interaction, molecular markers should be valuable for race identification at both the individual and population levels. For such a purpose, a genetic map of $L$. maculans is currently being constructed to identify markers linked to AvrLml (6). However, as the ultimate marker of race in natural populations is the Avr gene itself, one progeny of cross 23, carrying both AvrLm1 and AvrLm4, was chosen to construct a Bacterial Artificial Library and initiate map-based cloning of Avr genes (4).

\section{ACKNOWLEDGMENTS}

This work was supported by EU contract FAIR3CT96-1669 and grants from the "Société interprofessionnelle des oléagineux, protéagineux et cultures textiles" (SIDO) and "Agence de l'environnement et de la maîtrise de l'énergie" (ADEME). A. Attard was funded by the European Union. We thank IMASCORE partners, i.e., J. S. Dias (ISA, Lisbon, Portugal), B. Koopman (Univ. Göttingen, Germany), B. Fitt (IACR, Rothamsted, UK), A. Penaud (CETIOM, Grignon, France), M. Jedryzcka (IGR, Poznan, Poland), H. Busch (DSV, Salzkotten, Germany), and S. Veillet (Monsanto SAS, Toury, France) for devising the novel rating scale used here, and J. West (Plant Pathology, IACR-Rothamsted, UK) for reviewing the manuscript.

\section{LITERATURE CITED}

1. Ansan-Melayah, D., Balesdent, M.-H., Buée, M., and Rouxel, T. 1995. Genetic characterization of AvrLm1, the first avirulence gene of Leptosphaeria maculans. Phytopathology 85:1525-1529.

2. Ansan-Melayah, D., Balesdent, M.-H., Delourme, R., Pilet, M. L., Tanguy, X., Renard, M., and Rouxel, T. 1998. Genes for race-specific resistance against blackleg disease in Brassica napus L. Plant Breed.
117:373-378.

3. Ansan-Melayah, D., Rouxel, T., Bertrandy, J., Letarnec, B., MendesPereira, E., and Balesdent, M. H. 1997. Field efficiency of Brassica napus specific resistance correlates with Leptosphaeria maculans population structure. Eur. J. Plant Pathol. 103:835-841.

4. Attard, A., Gourgues, M., Billault, A., Soravito, C., Balesdent, M. H., and Rouxel, T. 1999. Map-based cloning of avirulence genes in the ascomycete Leptosphaeria maculans. Construction and screening of a bacterial artificial chromosome (BAC) library. Page 46 in: Molecular Genetics of Plant-Microbe Interactions. Proc. Conf. Int. Cong. Mol. Genet. Plant-Microbe Interact., 9th. The American Phytopathological Society, St. Paul, MN.

5. Badawy, H. M. A., Hoppe, H. H., and Koch, E. 1991. Differential reactions between the genus Brassica and aggressive single spore isolates of Leptosphaeria maculans. J. Phytopathol. 131:109-119.

6. Balesdent, M. H., Attard, A., Gourgues, M., Olivier-Kuhn, M. L., and Rouxel, T. 1999. Map-based cloning of avirulence genes in the ascomycete Leptosphaeria maculans. Genetic linkage map and molecular markers linked to the AvrLm1 locus. Page 46 in: Molecular Genetics of Plant-Microbe Interactions. Proc. Conf. Int. Cong. Mol. Genet. PlantMicrobe Interact., 9th. The American Phytopathological Society, St. Paul, MN.

7. Balesdent, M. H., Desthieux, I., Gall, C., Robin, P., and Rouxel, T. 1995 Quantification of Leptosphaeria maculans growth in cotyledons of Brassica napus using ELISA. J. Phytopathol. 143:65-73.

8. Balesdent, M. H., Gall, C., Robin, P., and Rouxel, T. 1992. Intraspecific variation in soluble mycelial protein and esterase patterns of Leptosphaeria maculans French isolates. Mycol. Res. 96:677-684.

9. CETIOM. 1998. Colza d'hiver: Les techniques culturales, le contexte économique. Edition CETIOM, Paris, France.

10. Delwiche, P. A. 1980. Genetic aspects of blackleg (Leptosphaeria maculans) resistance in rapeseed (Brassica napus). Ph.D. diss., University of Wisconsin, Madison.

11. Ferreira, M. E., Rimmer, S. R., Williams, P. H., and Osborn, T. C. 1995. Mapping loci controlling Brassica napus resistance to Leptosphaeria maculans under different screening conditions. Phytopathology 85:213217.

12. Fitt, B. D. L., Gladders, P., Turner, J. A., Sutherland, K. G., Welham, S. J., and Davies, J. M. L. 1997. Prospects for developing a forecasting scheme to optimize use of fungicides for disease control on winter oilseed rape in the UK. Aspects Appl. Biol. 48:135-142.

13. Foisset, N., Delourme, R., Barret, P., and Renard, M. 1995. Molecular tagging of the dwarf BREIZH (Bzh) gene in Brassica napus. Theor. Appl. Genet. 91:756-761.

14. Gall, C., Balesdent, M.-H., Desthieux, I., Robin, P., and Rouxel, T. 1995. Polymorphism of $\operatorname{Tox}^{0}$ Leptosphaeria maculans isolates as revealed by soluble protein and isozyme electrophoresis. Mycol. Res. 99:221-229.

15. Gall, C., Balesdent, M.-H., Robin, P., and Rouxel, T. 1994. Tetrad analysis of acid phosphatase, soluble protein patterns, and mating type in Leptosphaeria maculans. Phytopathology 84:1299-1305.

16. Gugel, R. K., and Petrie, G. A. 1992. History, occurrence, impact, and control of blackleg of rapeseed. Can. J. Plant Pathol. 14:36-45.

17. Hammond, K. E., Lewis, B. G., and Musa, T. M. 1985. A systemic pathway in the infection of oilseed rape plants by Leptosphaeria maculans. Plant Pathol. 34:557-565.

18. Hoppe, H. H., Kuswinanti, T., and Sock, J. 1994. Pathotypen von Phoma lingam. Proc. Rapssymposium zu fragen de Phytopathologie und des Pflanzenschutzes, Universität Rostock.

19. Koch, E., Song, K., Osborn, T. C., and Williams, P. H. 1991. Relationship between pathogenicity and phylogeny based on restriction fragment length polymorphism in Leptosphaeria maculans. Mol. PlantMicrobe Interact. 4:341-349.

20. Kuswinanti, T., Sock, J., and Hoppe, H. H. 1995. Variation in virulence of aggressive isolates of Leptosphaeria maculans based on cotyledon reactions on an extended differential set. Pages 1248-1250 in. Proc. Int. Rapeseed Cong., 9th. Henry Ling Ltd., Dorchester, United Kingdom.

21. Kutcher, H. R., van den Berg, C. G. J., and Rimmer, S. R. 1993. Variation in pathogenicity of Leptosphaeria maculans on Brassica spp. based on cotyledon and stem reactions. Can. J. Plant Pathol. 15:253-258.

22. Leclair, S., Ansan-Melayah, D., Rouxel, T., and Balesdent, M. H. 1996. Meiotic behavior of the minichromosome in the phytopathogenic ascomycete Leptosphaeria maculans. Curr. Genet. 30:541-548.

23. Mahuku, G. S., Goodwin, P. H., Hall, R., and Hsiang, T. 1997. Variability in the highly virulent type of Leptosphaeria maculans within and between oilseed rape fields. Can. J. Bot. 75:1485-1492.

24. Mayerhofer, R., Bansal, V. K., Thiagarajah, M. R., Stringam, G. R., and Good, A. G. 1997. Molecular mapping of resistance to Leptosphaeria maculans in Australian cultivars of Brassica napus. Genome 40:294-301.

25. McNabb, W. M., van den Berg, C. G. J., and Rimmer, S. R. 1993. Comparison of inoculation methods for selection of plants resistant to Lep- 
tosphaeria maculans in Brassica napus. Can. J. Plant Sci. 73:1199-1207.

26. Mengistu, A., Rimmer, S. R., Koch, E., and Williams, P. H. 1991. Pathogenicity grouping of isolates of Leptosphaeria maculans on Brassica napus cultivars and their disease reaction profiles on rapid-cycling Brassicas. Plant Dis. 75:1279-1282.

27. Pilet, M. L., Delourme, R., Foisset, N., and Renard, M. 1998. Identification of loci contributing to quantitative field resistance to blackleg disease, causal agent Leptosphaeria maculans (Desm.) Ces. et de Not., in winter rapeseed (Brassica napus L.). Theor. Appl. Genet. 96:23-30.

28. Pongam, P., Osborn, T. C., and Williams, P. H. 1998. Genetic analysis and identification of amplified fragment length polymorphism markers linked to the alm1 avirulence gene of Leptosphaeria maculans. Phytopathology 88:1068-1072.

29. Rouxel, T., and Séguin-Swartz, G. 1995. International blackleg of crucifers network. Blackleg News 5:15-16.

30. Salisbury, P. A., Ballinger, D. J., Wratten, N., Plummer, K. M., and Howlett, B. J. 1995. Blackleg disease on oilseed Brassica in Australia: A review. Aust. J. Exp. Agric. 35:665-672.

31. Volke, B. 1999. Leptosphaeria maculans, der Erreger der Wurzelhalsund Stengelfäule an Raps: Verbreitung verschiedener Pathogenitätsgruppen in Europa, Quantifizierung des Befalls und Shadwirkung im Freiland. Ph.D. thesis, University of Göttingen, Germany.

32. Waalwijk, C., Mendes, O., Bonants, P., Verstappen, E., and Kema, G 1999. Map-based cloning of a (complex) avirulence locus in $M y$ cosphaerella graminicola. Page 53 in: Molecular Genetics of Plant-Microbe Interactions. Proc. Conf. Int. Cong. Mol. Genet. Plant-Microbe Interact., 9th. The American Phytopathological Society, St. Paul, MN.

33. Whisson, S. C., Drenth, D., Maclean, J., and Irwin, J. A. G. 1997. Phytophthora sojae avirulence genes, RAPD, and RFLP markers used to construct a detailed genetic linkage map. Mol. Plant-Microbe Interact. 8:988-995.

34. Williams, P. H., and Delwiche, P. A. 1979. Screening for resistance to blackleg of crucifers in the seedling stage. Pages 164-170 in: Proc. Eucarpia Conf. Breeding Cruciferous Crops. Wageningen, the Netherlands. 